\title{
Brief History of Ultra-light Scalar Dark Matter Models
}

\author{
Jae-Weon Lee Le $^{1, \star}$ \\ ${ }^{1}$ Department of renewable energy, Jungwon university, 85 Munmu-ro, Goesan-eup, Goesan-gun, Chungcheongbuk-do, 367-805, \\ Korea
}

\begin{abstract}
This is a review on the brief history of the scalar field dark matter model also known as fuzzy dark matter, BEC dark matter, wave dark matter, or ultra-light axion. In this model ultra-light scalar dark matter particles with mass $m=O\left(10^{-22}\right) \mathrm{eV}$ condense in a single Bose-Einstein condensate state and behave collectively like a classical wave. Galactic dark matter halos can be described as a self-gravitating coherent scalar field configuration called boson stars. At the scale larger than galaxies the dark matter acts like cold dark matter, while below the scale quantum pressure from the uncertainty principle suppresses the smaller structure formation so that it can resolve the small scale crisis of the conventional cold dark matter model.
\end{abstract}

Despite long efforts dark matter (DM) remains a great mystery in physics and astronomy [1]. While numerical results of the cold dark matter (CDM) model are remarkably successful in explaining the large scale structure of the universe, it encounters some problems at the scale of galactic or sub-galactic structures. For example, the numerical simulations usually predict cusped central halo density and too many sub-halos and small galaxies, which seem to be in contradiction with observations [2-5].

Recently, there is a growing interest in the idea that DM particles are ultra-light scalars in Bose-Einstein condensate (BEC). (For a review, see Refs. 6-13) In this model, due to the tiny DM particle mass $m=O\left(10^{-22}\right) \mathrm{eV}$, the DM particle number density is very high and hence the inter-particle distance is much smaller than the de Broglie wave length of the DM particles. Therefore, the particles are in BEC and move collectively as a wave rather than incoherent particles. At the scale larger than galaxies the DM perturbation behaves like that of CDM, while below the scale quantum pressure from the uncertainty principle suppresses the small structure formation, which makes it a viable alternative to CDM. This property helps us to resolve the small scale problems of the CDM model such as the missing satellite problem or the cusp/core problem [14].

Before 2000 there were only a few groups of scientists working on this topic, without much communication even between them. Being unaware of precedent works, many researchers in this field independently proposed similar ideas with various names such as BEC DM, scalar field DM (SFDM), fuzzy DM, ultra-light axion (ULA), ultralight axion like particle (ALP), wave DM, $\psi$ DM, repulsive DM or (super)fluid DM among many, although the basic physics of these models is quite similar. (Henceforth, I will use the term 'SFDM' for the model.) This unfortunate situation has brought some confusions among researchers in this field. Therefore, at this point, it is desirable to summarize what have already attempted in this exciting field. 1

The hypothesis that galactic DMs are ultra-light scalar particles in BEC has a long history. In Ref. [15] Baldeschi et al. considered the galactic halo model of self-gravitating bosons with mass $m \simeq 10^{-24} \mathrm{eV}$, which was obtained by comparing the de Broglie wave length of DM to the typical galaxy size. In [16] Membrado et al. calculated the rotation curve of self-gravitating boson halos using the ground state of the bosons, which later turned out to be adequate for dwarf galaxies [17]. In [18] Sin suggested that the halos are like giant atoms made of ultra light BEC DM particles such as pseudo Nambu-Goldstone boson (PNGB). $\mathrm{He}$ tried to explain the observed flat rotation curves (RCs) using the excited states of the BEC DM of the Non-linear Schrödinger equation and emphasized the ripple structures of the RCs as a smoking gun of the theory. The particle mass $m \simeq 3 \times 10^{-23} \mathrm{eV}$ was first obtained by fitting the observed RC of galaxy NGC2998. (See also [19, 20]) Following his work, Lee and Koh [21,22] suggested that DM halos are giant boson stars (BSs) which can be described by the relativistic scalar field theory with gravity, i.e., Einstein Klein-Gordon equation (EKG). In this model DM particles are represented by scalar field $\phi$ with a typical action

$$
S=\int \sqrt{-g} d^{4} x\left[\frac{-R}{16 \pi G}-\frac{g^{\mu \nu}}{2} \phi_{; \mu}^{*} \phi_{; v}-U(\phi)\right],
$$

where the potential $U(\phi)=\frac{m^{2}}{2}|\phi|^{2}+\frac{\lambda}{4}|\phi|^{4}$ was considered as an example. This model can be reduced to the BEC model in the Newtonian limit. The field is often assumed to be complex but can be real and have more general potentials. From the maximum stable central density and

\footnotetext{
^e-mail: scikid@jwu.ac.kr
}

\footnotetext{
${ }^{1}$ The list in the references of this paper is by no means exhaustive.
} 
the maximum halo mass $\left(M_{h} \simeq 10^{12} M_{\odot}\right)$ the mass bound $10^{-28} \leq m \leq 10^{-22} \mathrm{eV}$ was obtained for $\lambda=0$ case (now often called fuzzy DM) from the BS theory. It was emphasized that the repulsive self-interaction among DM particles, if there is, provides an additional pressure against the gravitational collapse and drastically changes the length scale to $O\left(\sqrt{\lambda} m_{P} / m^{2}\right)$ even for a tiny $\lambda$, where $m_{P}$ is the planck mass. An approximate analytic solution of KGE, $|\phi|^{2} \sim \sin (k r) / k r$, was obtained for $\Lambda \equiv \lambda m_{P}^{2} / m^{2} \gg 1$ (Thomas-Fermi limt) [21, 23]. If $\lambda<0$ (as in the Taylorexpanded potential for ULA) the halo can be unstable [21] as argued in [24].

On the other hand Press et al. [25], consider a 'soft boson' with somewhat longer Compton wavelength $\lambda_{c}=$ $30 k p c$ than that for the typical SFDM. Widrow and Kaiser [26] proposed a numerical technique for the evolution of collisionless matter using the Schrödinger-Poisson equations (SPE).

Similar ideas were independently investigated by many authors [23, 26-40, 40-45]. For example, a model with $m=0$ was suggested in [27], which has a stability issue. The SFDM without self-interaction (i.e., Fuzzy DM) with $m \simeq 10^{-22} \mathrm{eV}$ was shown to be able to solve the small scale issues [31]. Guzman and Matos et al. [46, 47] extensively studied various astrophysical aspects of the SFDM. Other attempts are based, for example, on a non-minimal coupling [28], quintessence [29, 48], Repulsive DM [30], fluid DM [32], nontopological soliton [33], cosh potential $[35,36]$, and ULA $[34,37,38]$ among many [39$44,49]$. The BEC nature of this DM was extensively studied in $[9,23,50]$ by Harko, Chavanis and their colleagues. More recently cosmology of the ULA is thoroughly studied in $[51,52]$ by Marsh et al. The ULA has usually a cosine potential, which is often approximated to be a quadratic potential. In that case the calculation results are almost same to those of the fuzzy DM. Other works are described in the followings.

In the SFDM model the macroscopic wave function of the halo DM is often described by the following SPE,

$$
\begin{aligned}
i \hbar \partial_{t} \psi & =-\frac{\hbar^{2}}{2 m} \nabla^{2} \psi+m V \psi \\
\nabla^{2} V & =4 \pi G \rho_{d}
\end{aligned}
$$

which can be derived from the EKG for weak gravity [53] using $\psi=\sqrt{m} \phi$ or from the many-body formalism. Here, the DM density is $\rho_{d}=m|\psi|^{2}=m^{2}|\phi|^{2}$ and $V$ is the self-gravitation potential. Once we get $\psi$ from the SPE we can predict the astrophysical observables. For example, for a spherical halo the rotation velocity at radius $r$ is $v_{\text {rot }}(r)=\sqrt{\frac{G M(r)}{r}}$, where $M(r)=4 \pi \int_{0}^{r} r^{\prime 2} \rho_{d}\left(r^{\prime}\right) d r^{\prime}$ is the mass within $r$.

To study the cosmological structure formation in the fluid approach, it is useful to reduce the Schrödinger equation to the Madelung euations [7, 10] using

$$
\psi(r, t)=\sqrt{\rho(r, t)} e^{i S(r, t)} .
$$

Substituting Eq. (3) into the Schrödinger equation gives a continuity equation

$$
\frac{\partial \rho}{\partial t}+\nabla \cdot(\rho \mathbf{v})=0
$$

and an Euler-like equation

$$
\frac{\partial \mathbf{v}}{\partial t}+(\mathbf{v} \cdot \nabla) \mathbf{v}+\nabla V+\frac{\nabla p}{\rho}-\frac{\nabla Q}{m}=0
$$

with a quantum potential $Q \equiv \frac{\hbar^{2}}{2 m} \frac{\Delta \sqrt{\rho}}{\sqrt{\rho}}$, a fluid velocity $\mathbf{v} \equiv \nabla S / 2 m$, and the pressure $p$ from a self-interaction (if any). The quantum pressure term $\nabla Q / m$ is absent in the CDM models. We have ignored the cosmic expansion for simplicity. Perturbing the above equations around $\rho=\bar{\rho}$, $\mathbf{v}=0$, and $V=0$ gives an equation for density perturbation $\delta \rho \equiv \rho-\bar{\rho}$,

$$
\frac{\partial^{2} \delta \rho}{\partial t^{2}}+\frac{\hbar^{2}}{4 m^{2}} \nabla^{2}\left(\nabla^{2} \delta \rho\right)-c_{s}^{2} \nabla^{2} \delta \rho-4 \pi G \bar{\rho} \delta \rho=0,
$$

where $c_{s}$ is the classical sound velocity from $p$, and $\bar{\rho}$ is the average background matter density (See, for example, Refs. 50, 54 for details.).

The Fourier transformed equation of the density contrast $\delta \equiv \delta \rho / \bar{\rho}=\sum_{k} \delta_{k} e^{i k \cdot r}$ with a wave vector $k$ is

$$
\frac{d^{2} \delta_{k}}{d t^{2}}+\left[\left(c_{q}^{2}+c_{s}^{2}\right) k^{2}-4 \pi G \bar{\rho}\right] \delta_{k}=0,
$$

where $c_{q}=\hbar k / 2 m$ is a quantum velocity. For a small $k$ (at a large scale) the $c_{s}$ dependent term dominates and SFDM behaves like the CDM (with pressure) while for a large $k$ (at a small scale) the $c_{q}$ dependent term dominates and quantum pressure prevents the structure formation [31, 55-57]. This interesting behavior of the SFDM is confirmed by the precise numerical studies [58] and makes the SFDM an ideal alternative for the CDM. This property alleviates the small scale problems of the CDM model $[32,35,56,59]$. Equating $c_{q}^{2} k^{2}$ with $4 \pi G \bar{\rho}$ gives the quantum Jeans length scale $[31,60,61]$ at the redshift $z$,

$$
\lambda_{Q}(z)=\frac{2 \pi}{k}=\left(\frac{\pi^{3} \hbar^{2}}{m^{2} G \bar{\rho}(z)}\right)^{1 / 4}
$$

which leads to the minimum length scale and the mass scale of galactic halos formed at $z$ [62-64].

Dwarf galaxies are the smallest dark-matter-dominated objects and, hence, are ideal objects for understanding the nature of dark matter. The Jeans length above provides a natural cut-off in the mass power spectrum preventing the overproduction of subhalos and the dwarf satellite galaxies $[31,32,35,56,59,65]$. The SFDM could solve the cusp problem $[17,31,32,32,66,66-68]$ because of the boundary condition for the SPE at the halo center. There are many works explaining the rotation curves of dwarf [17, 23, 69], and large galaxies [29, 43, 70-78] in this model. By fitting RCs one can obtain the scalar field mass $m \sim 10^{-22} \mathrm{eV}$, and interestingly the SFDM with this mass range can resolve many of the small scale problems of CDM models. One exception is the Lyman alpha tension $[79,80]$ which is under debate [81]. This 
model can be used to explain the puzzling minimum mass scale $[63,64]$ and the minimum length scale of galaxies [82, 83] regardless of their luminosity [84] and the observed size evolution [62] of the most massive galaxies [85-87].

The rotating ellipsoidal gravitational potentials of the SFDM halos was shown to induce spiral arms and shell structures in the visible matter of galaxies [44]. The effect of embedding a supermassive black hole in a SFDM halo was considered in [88]. It was also argued that this model can explain the M-sigma relation of supermassive black holes [89].

It is suggested that the SFDM can explain the contradictory behaviors of DM in collisions of galaxy clusters [90-94]. For a fast collision as observed in the Bullet cluster two dark matter halos pass each other in a solitonlike way, while for a slow collision as observed in the Abell 520 the halos merge due to the wave nature of the SFDM. This idea might explain the origin of the dark galaxy and the galaxy without dark matter [90] .

The SFDM model reproduces the evolution of the cosmological densities [95] and the spectrum of the cosmic microwave background $[37,96]$ if $m>10^{-24} \mathrm{eV}$.

The typical QCD axion has some difficulties to be a SFDM. It has $\cos (\phi)$ potential which is effectively attractive to $O\left(\phi^{4}\right)$, and its mass $m_{a} \simeq 10^{-5} \mathrm{eV}$ is too heavy, and it is a real field giving oscillatons instead of boson stars [21]. On the other hand ultra-light axion (ULA) with mass $m_{a} \simeq 10^{-22} \mathrm{eV}$ recently attracts much interest, but if we consider only the quadratic potential for ULA, ULA is almost identical to the typical SFDM or Fuzzy DM. Therefore, we can use the cosmological constraints of ULA for SFDM and vice versa in many situations. There is an idea that a vector field in a modified gravity action could be identified with a BEC [97]. SFDM can be dark energy [98-101] and have quantum entanglement [102].

It is also suggested that the time dependent potential induced by oscillating SFDM influences the pulse arrival residual which can be observed by the Square Kilometre Array (SKA) experiment [103, 104] or by laser interferometer gravitational wave detectors [105]. The oscillation of SFDM might resonate with binary pulsars [106].

In summary, SFDM with mass about $10^{-22} \mathrm{eV}$ satisfies many cosmological constraints [107] and seems to be a viable alternative to CDM. Therefore, this model deserves careful considerations. Especially, it is desirable to find a good particle physics model for SFDM [13, 108].

\section{acknowledgments}

This work was supported by the Jungwon University Research Grant (2016-040).

\section{References}

[1] J. Silk, Challenges in Cosmology from the Big Bang to Dark Energy, Dark Matter and Galaxy Formation, in 14th International Symposium on Nuclei in the Cosmos (NIC-XIV) Niigata, Japan, June 19-24, 2016 (2016), 1611.09846
[2] P. Salucci, F. Walter, A. Borriello, Astron. Astrophys. 409, 53 (2003), astro-ph/0206304

[3] J.F. Navarro, C.S. Frenk, S.D.M. White, Astrophys. J. 462, 563 (1996)

[4] W.J.G. de Blok, A. Bosma, S.S. McGaugh, astro$\mathrm{ph} / 0212102$ (2002)

[5] A. Tasitsiomi, International Journal of Modern Physics D 12, 1157 (2003)

[6] J.W. Lee, Journal of Korean Physical Society 54, 2622 (2009), 0801.1442

[7] A. Suárez, V.H. Robles, T. Matos, Astrophysics and Space Science Proceedings 38, 107 (2014), 1302.0903

[8] T. Rindler-Daller, P.R. Shapiro, Modern Physics Letters A 29, 1430002 (2014), 1312. 1734

[9] T. Harko, Phys. Rev. D 89, 084040 (2014), 1403.3358

[10] P.H. Chavanis, Phys. Rev. D 84, 043531 (2011), 1103.2050

[11] K. Huang, C. Xiong, X. Zhao, International Journal of Modern Physics A 29, 1450074 (2014), 1304.1595

[12] D.J.E. Marsh, Phys. Rept. 643, 1 (2016), 1510.07633

[13] L. Hui, J.P. Ostriker, S. Tremaine, E. Witten (2016), 1610.08297

[14] J.R. Primack, New Journal of Physics 11, 105029 (2009), 0909. 2247

[15] M.R. Baldeschi, G.B. Gelmini, R. Ruffini, Physics Letters B 122, 221 (1983)

[16] M. Membrado, A.F. Pacheco, J. Sañudo, Phys. Rev. A 39, 4207 (1989)

[17] T. Matos, D. Nunez, astro-ph/0303455 (2003), astro-ph/0303455

[18] S.J. Sin, Phys. Rev. D50, 3650 (1994), hep-ph/9205208

[19] S.U. Ji, S.J. Sin, Phys. Rev. D 50, 3655 (1994)

[20] C. Lee, S. Sin, J.Korean Phys.Soc. 28, 16 (1995)

[21] J.W. Lee, I.G. Koh, Phys. Rev. D53, 2236 (1996), hep-ph/9507385

[22] J.W. Lee, I.G. Koh, Galactic halo as a soliton star , Abstracts, bulletin of the Korean Physical Society, 10 (2) (1992)

[23] C.G. Boehmer, T. Harko, JCAP 0706, 025 (2007), 0705.4158

[24] A.H. Guth, M.P. Hertzberg, C. Prescod-Weinstein, Phys. Rev. D92, 103513 (2015), 1412.5930

[25] W.H. Press, B.S. Ryden, D.N. Spergel, Physical Review Letters 64, 1084 (1990)

[26] L.M. Widrow, N. Kaiser, Astrophys. J. Lett. 416, L71 (1993)

[27] F.E. Schunck, astro-ph/9802258 (1998)

[28] U. Nucamendi, M. Salgado, D. Sudarsky, Phys. Rev. Lett. 84, 3037 (2000), gr-qc/0002001

[29] A. Arbey, J. Lesgourgues, P. Salati, Phys. Rev. D 64, $123528(2001)$ 
[30] J. Goodman, New Astronomy Reviews 5, 103 (2000)

[31] W. Hu, R. Barkana, A. Gruzinov, Phys. Rev. Lett. 85, $1158(2000)$

[32] P. Peebles, Astrophys. J. 534, L127 (2000)

[33] E.W. Mielke, F.E. Schunck, Phys. Rev. D 66, 023503 (2002)

[34] E.W. Mielke, J.A.V. Perez, Physics Letters B 671, $174(2009)$

[35] V. Sahni, L. Wang, Phys. Rev. D 62, 103517 (2000)

[36] M. Alcubierre, F.S. Guzman, T. Matos, D. Nunez, L.A. Urena-Lopez, P. Wiederhold, Class. Quant. Grav. 19, 5017 (2002), gr-qc/0110102

[37] C.G. Park, J.c. Hwang, H. Noh, Phys. Rev. D 86, 083535 (2012), 1207.3124

[38] P. Sikivie, Q. Yang, Physical Review Letters 103, 111301 (2009), 0901. 1106

[39] B. Fuchs, E.W. Mielke, Mon. Not. Roy. Astron. Soc. 350, 707 (2004), astro-ph/0401575

[40] T. Matos, F.S. Guzman, L.A. Urena-Lopez, D. Nunez, astro-ph/0102419 (2001)

[41] M.P. Silverman, R.L. Mallett, Classical and Quantum Gravity 18, L103 (2001)

[42] U. Nucamendi, M. Salgado, D. Sudarsky, Phys. Rev. D 63, 125016 (2001)

[43] A.A. Julien Lesgourgues, P. Salati, New Astronomy Reviews 46, 791 (2002)

[44] H.L. Bray, ArXiv:1212.5745 (2012), 1212.5745

[45] J. Eby, C. Kouvaris, N.G. Nielsen, L.C.R. Wijewardhana, arXiv:1511.04474 (2015)

[46] T. Matos, F.S. Guzman, Class. Quant. Grav. 17, L9 (2000), gr-qc/9810028

[47] F.S. Guzman, T. Matos, H. Villegas-Brena, Rev. Mex. Astron. Astrofis. 37, 63 (2001), astro-ph/9811143

[48] A. Arbey, J. Lesgourgues, P. Salati, Phys. Rev. D 65, 083514 (2002)

[49] V. Sahni, L.M. Wang, Phys. Rev. D62, 103517 (2000), astro-ph/9910097

[50] P.H. Chavanis, Astronomy and Astrophysics 537, A127 (2012), 1103.2698

[51] D.J.E. Marsh, P.G. Ferreira, Phys. Rev. D82, 103528 (2010), 1009.3501

[52] R. Hlozek, D. Grin, D.J.E. Marsh, P.G. Ferreira, Phys. Rev. D91, 103512 (2015), 1410. 2896

[53] R. Friedberg, T.D. Lee, Y. Pang, Phys. Rev. D 35, 3640 (1987)

[54] A. Suarez, T. Matos, Mon. Not. Roy. Astron. Soc. 416, 87 (2011), 1101. 4039

[55] M. Alcubierre, F.S. Guzman, T. Matos, D. Nunez, L.A. Urena-Lopez, P. Wiederhold, Scalar field dark matter and galaxy formation, in Dark matter in astro- and particle physics. Proceedings, 4th Heidelberg International Conference, DARK 2002, Cape Town, South Africa, February 4-9, 2002 (2002)
[56] T. Matos, L. Arturo Ureña López, Phys. Rev. D 63, 063506 (2001)

[57] T. Harko, Mon. Not. Roy. Astron. Soc. 413, 3095 (2011), 1101.3655

[58] H.Y. Schive, T. Chiueh, T. Broadhurst, Nature Physics 10, 496 (2014), 1406.6586

[59] T. Matos, L.A. Urena-Lopez, Class. Quant. Grav. 17, L75 (2000)

[60] M.I. Khlopov, B.A. Malomed, I.B. Zeldovich, Mon. Not. Roy. Astron. Soc. 215, 575 (1985)

[61] D. Grasso, Phys.Rev. D41, 2998 (1990)

[62] J.W. Lee, Phys. Lett. B681, 118 (2009), 0805 . 2877

[63] J.W. Lee, Phys. Lett. B756, 166 (2016), 1511.06611

[64] J.W. Lee, S. Lim, JCAP 1001, 007 (2010), Q812. 1342

[65] A. Suárez, T. Matos, Mon. Not. Roy. Astron. Soc. 416, 87 (2011), 1101.4039

[66] A. Riotto, I. Tkachev, Phys. Lett. B484, 177 (2000), astro-ph/0003388

[67] K.Y. Su, P. Chen, JCAP 1108, 016 (2011), 1008.3717

[68] T. Matos, L.A. Urena-Lopez, Gen. Rel. Grav. 39, 1279 (2007)

[69] A. Arbey, J. Lesgourgues, P. Salati, Phys. Rev. D 68, $023511(2003)$

[70] J. Lesgourgues, A. Arbey, P. Salati, New Astronomy Reviews 46, 791 (2002)

[71] V.H. Robles, T. Matos, Mon. Not. Roy. Astron. Soc. 422, 282 (2012), 1201. 3032

[72] H.Y. Schive, M.H. Liao, T.P. Woo, S.K. Wong, T. Chiueh, T. Broadhurst, W.Y.P. Hwang, Phys. Rev. Lett. 113, 261302 (2014), 1407.7762

[73] T. Harko, Mon. Not. Roy. Astron. Soc. 413, 3095 (2011), 1101.3655

[74] V.H. Robles, T. Matos, Mon. Not. Roy. Astron. Soc. 422, 282 (2012), 1201. 3032

[75] T. Bernal, L.M. Fernández-Hernández, T. Matos, M.A. Rodríguez-Meza, ArXiv e-prints (2017), 1701.00912

[76] F.S. Guzman, T. Matos, Class. Quant. Grav. 17, L9 (2000)

[77] J.P. Mbelek, Astron. Astrophys. 424, 761 (2004), gr-qc/0411104

[78] T.H. Lee, B.J. Lee, Phys. Rev. D 69, 127502 (2004)

[79] V. Iršič, M. Viel, M.G. Haehnelt, J.S. Bolton, G.D. Becker, Phys. Rev. Lett. 119, 031302 (2017), 1703.04683

[80] E. Armengaud, N. Palanque-Delabrouille, D.J.E. Marsh, J. Baur, C. Yeche, Mon. Not. Roy. Astron. Soc. (2017), 1703.09126

[81] J. Zhang, J.L. Kuo, H. Liu, Y.L.S. Tsai, K. Cheung, M.C. Chu (2017), 1708.04389

[82] M. Mateo, Ann. Rev. Astron. Astrophys. 36, 435 (1998), astro-ph/9810070

[83] G. Gilmore, D. Zucker, M. Wilkinson, R.F.G. Wyse, V. Belokurov, J. Kleyna, A. Koch, N.W. Evans, E.K. 
Grebel, eprint:arXiv.org:0804.1919 (2008)

[84] L.E. Strigari, J.S. Bullock, M. Kaplinghat, J.D. Simon, M. Geha, B. Willman, M.G. Walker, Nature 454, 1096 (2008), 0808. 3772

[85] E. Daddi et al., Astrophys. J. 631, L13 (2005), astro-ph/0507504

[86] P.G. van Dokkum, M. Kriek, M. Franx, Nature 460, 717 (2009), 0906. 2778

[87] I. Trujillo, C.J. Conselice, K. Bundy, M.C. Cooper, P. Eisenhardt, R.S. Ellis, 382, 109 (2007)

[88] L.A. Urena-Lopez, A.R. Liddle, Phys. Rev. D66, 083005 (2002), astro-ph/0207493

[89] J.W. Lee, J. Lee, H.C. Kim, arXiv:1512.02351 (2015)

[90] J.W. Lee, S. Lim, D. Choi, arXiv:0805.3827 (2008)

[91] D. Castaneda Valle, E.W. Mielke, Annals Phys. 336, 245 (2013)

[92] A. Paredes, H. Michinel, Phys. Dark Univ. 12, 50 (2016), 1512.05121

[93] B. Schwabe, J.C. Niemeyer, J.F. Engels, Phys. Rev. D94, 043513 (2016), 1606.05151

[94] F.S. Guzman, J.A. Gonzalez, J.P. Cruz-Perez, Phys. Rev. D93, 103535 (2016), 1605 . 04856

[95] T. Matos, A. Vázquez-González, J. Magaña, Mon. Not. Roy. Astron. Soc. 393, 1359 (2009),
0806.0683

[96] I. Rodríguez-Montoya, J. Magaña, T. Matos, A. Pérez-Lorenzana, Astrophys. J. 721, 1509 (2010), 0908.0054

[97] J.W. Moffat, astro-ph/0602607 (2006)

[98] A. Arbey, J. Lesgourgues, P. Salati, Phys. Rev. D64, 123528 (2001), astro-ph/0105564

[99] T. Matos, arXiv:0909.3634 (2009), 0909. 3634

[100] K. Huang, Int. J. Mod. Phys. A28, 1330049 (2013), [,15(2014)], 1309.5707

[101] M. Gogberashvili, A.S. Sakharov, arXiv (2017), 1702.05757

[102] J.W. Lee, arXiv:1510.07968 (2015), 1510.07968

[103] A. Khmelnitsky, V. Rubakov, JCAP 1402, 019 (2014), 1309. 5888

[104] A. Aoki, J. Soda, Phys. Rev. D93, 083503 (2016), 1601.03904

[105] A. Aoki, J. Soda, arXiv:1608.05933 (2016)

[106] D. Blas, D.L. Nacir, S. Sibiryakov, Phys. Rev. Lett. 118, 261102 (2017), 1612.06789

[107] B. Li, T. Rindler-Daller, P.R. Shapiro, Phys. Rev. D89, 083536 (2014), 1310.6061

[108] J.E. Kim, D.J.E. Marsh, Phys. Rev. D93, 025027 (2016), 1510.01701 